\title{
Необходимость межлабораторных сличений в современной метрологии
}

\author{
А. Кривов, д. т. н. ${ }^{1}$, Е. Смирнова ${ }^{2}$, К. Бондин ${ }^{3}$, П. Николаев ${ }^{4}$
}

УДК 006.91:621.317.089.6 | ВАК 05.11.15

\begin{abstract}
Расширение задач, решаемых электроникой, и возрастание ее роли в важнейших сферах обеспечения жкизни общества вызывают объективную потребность в увеличении объемов измерений и повышении их точности. Метрологическое обеспечение современных электронных производств предполагает применение различных форм и способов обеспечения требуемой точности измерений (поверка средств измерений, аттестация методик измерений и др.).

В международной практике все большее значение приобретают технологии межлабораторных сравнительных испытаний (МСИ) - специалисты также называют их межлабораторными сличениями, - имеющие весомые потенциальные возможности. В данной статье рассматриваются некоторые вопросы реализации требований действующих стандартов в части, относящейся к МСИ, и приводятся краткие сведения о пилотном проекте по МСИ результатов калибровки и поверки электроизмерительных приборов, выполненном компанией "Диполь» в сотрудничестве с рядом предприятий из разных отраслей промышленности.
\end{abstract}

остояние сегодняшней прикладной метрологии является итогом многолетней деятельности и международного сотрудничества метрологов различных отраслей экономики. Поэтому для объяснения появившейся в последние годы потребности в развитии практики межлабораторных сравнительных испытаний надо обратиться к базовым положениям метрологии.

Отечественная концепция единства измерений или распространенное за рубежом понятие прослеживаемости измерений принимаются за основу метрологической практики. Совокупность метрологических работ реализует два основных постулата, в соответствии с которыми должны быть обеспечены:

- сходимость результатов многократных измерений одной и той же величины в одинаковых условиях и одинаковыми методами и средствами измерений;

- воспроизводимость результатов измерений одной и той же величины, полученных в разных местах и различными методами и средствами измерений.

AO «НПФ «Диполь», заместитель директора, ask@dipaul.ru. АО «НПФ "Диполь», инженер по метрологии и стандартизации, SmirnovaEA@dipaul.ru.

ООО «Профигрупп», генеральный директор, bondin@pg-spb.ru. О०० «Профигрупп», главный метролог, nikolaev@pg-spb.ru.
Выполнение обоих постулатов связано с большим количеством объективных и субъективных условий и факторов, соблюдение которых играет важную роль в метрологической практике. Сторонники развития МСИ, а это, главным образом, зарубежные специалисты, считают, что традиционные способы и процедуры проверки лабораторий в ходе их аккредитации не гарантируют выполнение всех условий. Даже в аккредитованных поверочных или калибровочных лабораториях могут возникнуть неучитываемые факторы, приводящие к ошибочным результатам.

мсИ как способ контроля качества метрологических работ практически неизвестен метрологам отечественных предприятий. На сайтах метрологических организаций можно найти единичные предложения по раундам сличений результатов калибровки средств измерений. Такая ситуация объясняется сложившейся практикой метрологического обеспечения промышленности. Прикладная метрология в нашей стране базируется на методологии обеспечения единства измерений как для сферы государственного регулирования, так и вне ее, например, при технологических измерениях. На практике полагается, что выполнение требований законодательства и базовых стандартов к основным метрологическим работам гарантирует необходимое качество результатов поверки и калибровки.

Такому подходу соответствует развитие контроля качества работ "по вертикали»: через аккредитацию, 
предоставление полномочий, отраслевой (корпоративный) аудит, государственный и внутренний метрологический надзор. Пригодность средств измерений обеспечивается передачей единицы величины по государственной поверочной схеме и "правильной" организацией метрологических работ в соответствии с разработанными стандартами предприятий. Влияние на качество поверки приборов и точность результатов измерений дополнительных факторов, связанных с "незначительными" отклонениями условий измерений, изменением характеристик средств измерений и эталонов в течение межповерочного интервала, методическими особенностями измерений, как правило, промышленными метрологами не оценивается. Апри организации метрологических работ и вовсе встречаются случаи несоответствия процедур поверки и калибровки установленным требованиям.

Современная прикладная метрология базируется на ключевых положениях международных стандартов в области менеджмента качества продукции и услуг, компетентности испытательных и калибровочных лабораторий, которые предусматривают полный количественный учет всех влияющих на точность измерений факторов. С этим подходом связан принцип метрологической прослеживаемости измерений как основного критерия признания их результатов, а также введение в практику учета и оценки всех составляющих неопределенности измерений. Следствием такого подхода является также развитие МсИ - эффективного способа проверки квалификации как испытательных, таки метрологических лабораторий. МСИ реализуют «горизонтальные» связи метрологов и позволяют получить объективные данные по экспериментальной проверке лабораторий, выявить опасные ситуации и выполнить корректирующие действия.

Потенциальные возможности МСИ привлекли внимание компании "Диполь" к этому новому виду метрологической деятельности начиная с 2015 года. За это время специалистами компании были рассмотрены вопросы реализации требований действующих стандартов к МСИ для определения качества поверки и калибровки измерительных приборов и других метрологических задач, выполнены пилотные проекты по межлабораторным сличениям результатов калибровки и поверки электроизмерительных приборов.

\section{СТАНДАРТЫ}

Развитие МСИ в зарубежных странах привело к созданию и распространению соответствующей нормативной базы. Согласно действующим стандартам, лаборатория должна располагать процедурами управления качеством и контролировать достоверность проведенных испытаний и калибровки, в том числе путем участия в МСИ. Такое требование о проведении МСИ как одного из основных способов контроля качества результатов испытаний и калибровки содержится в ГОСТ ИСО / МЭК 17025-2009, устанавливающем требования к компетентности испытательных и калибровочных лабораторий. В соответствии с ними к основным факторам, влияющим на правильность и достоверность результатов измерений, относятся:

- человеческий фактор;

- помещения и условия окружающей среды;

- методики измерений, испытаний и калибровки и оценка пригодности методик;

- оборудование;

- прослеживаемость измерений;

- отбор образцов;

- обращение с объектами измерений, испытаний и калибровки.

Влияние данных факторов на результаты измерений можно оценить с помощью межлабораторных сличительных испытаний

Требования к проведению МСИ содержатся также в критериях аккредитации в Национальной системе аккредитации, которые разработаны в соответствии с действующим законодательством РФ, и в Политике Росаккредитации по проверке квалификации калибровочных лабораторий путем МСИ. Казалось бы, это должно стать основным мотивом для развития мСИ в нашей стране, поскольку таким образом лаборатория получает дополнительное преимущество при прохождении процедуры аккредитации. Но в действительности роль МСИ в прикладных задачах метрологии гораздо шире, чем подтверждение компетентности при аккредитации. Авторы статьи придерживаются мнения о необходимости рассмотрения МСИ как доступного и объективного способа контроля результатов широкого спектра метрологических работ, позволяющего выявить не учитывавшиеся ранее факторы влияния.

Опыт организации и проведения проверки квалификации лабораторий на основе МСИ отражен в международном стандарте ГОСТ ISO/IEC17043-2013. Этот документ содержит общие требования к порядку проведения работ и требования к провайдеру (организатору) МсИ, варианты программ проверки квалификации и методы статистического анализа результатов сличений. Основные правила статистического анализа при МСИ, выбор приписанных значений контрольных образцов, критерии для интерпретации результатов измерений участников регламентированы в ГОСТ Р ИСО 13528-2010, который с 2019 года заменен гОСТ Р 50779.60-2017 (ИСО 13528:2015) «Статистические методы. Применение при проверке квалификации посредством межлабораторных испытаний». 


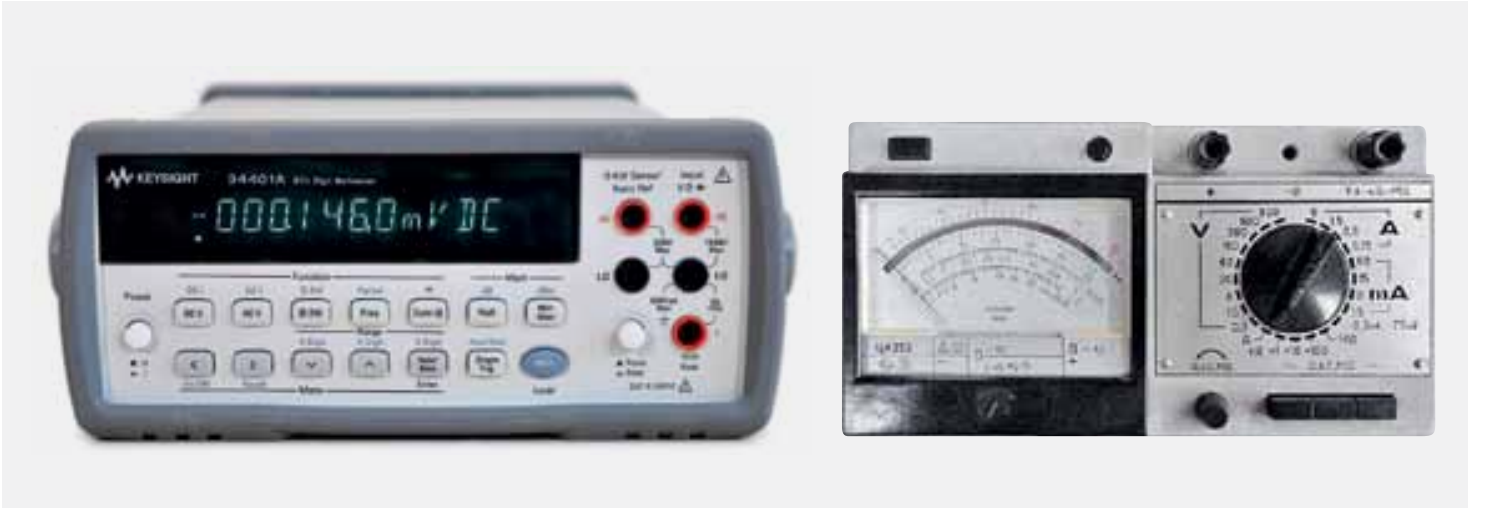

Рис. 1.

Электроизмерительные приборы контрольные образцы для межлабораторных сличений

\section{КРАТКАЯ ХАРАКТЕРИСТИКА РЕЗУЛЬТАТОВ ПИЛОТНЫХ МСИ}

Учитывая заинтересованность отечественных метрологов, специалисты компании "Диполь" организовали и совместно с предприятиями судостроительной, атомной и оборонной промышленности, железнодорожного транспорта провели несколько раундов МСИ результатов калибровки электроизмерительных приборов. Отсутствие опыта проведения МСИ для отечественных метрологических лабораторий определило пилотный характер проведенных сличений. В качестве контрольных образцов для проведения сличений были выбраны широко применяемые в промышленности электроизмерительные приборы: цифровой мультиметр типа 34401А фирмы Keysight и комбинированный прибор типа Ц4352 (рис. 1).

Сличения результатов калибровки этих приборов проводились по измерениям напряжения и силы постоянного и переменного тока. Для цифрового мультиметра проводились также измерения по электрическому сопротивлению и частоте. По мнению организаторов сличений, такой выбор контрольных образцов позволил в пилотном проекте, не отвлекаясь на изучение применяемых приборов, получить большой массив экспериментальных данных, сравнить результаты оценки квалификации для приборов с различным уровнем конструктивной сложности и показателей точности.
В группу участников межлабораторных сличений входили метрологические лаборатории семи крупных предприятий, аккредитованные или готовящиеся к аккредитации в области поверки и калибровки средств измерений. Для проведения сличений была использована стандартная последовательная программа МсИ результатов калибровки контрольного образца в лабораториях с контролем стабильности характеристик приборов за время проведения сличений в референтной лаборатории, в качестве которой была выбрана аккредитованная лаборатория петербургской компании ООО «Профигрупп". Схема проведения МСИ показана на рис. 2.

После проведения калибровки приборов и получения от участников сличений протоколов калибровки был проведен анализ результатов. Статистический анализ, в процессе которого происходит оценка собранной информации, является содержательной частью МсИ.

Статистический анализ данных включал в себя следующие процедуры:

- установление приписанныхзначений погрешности измерений и значений их расширенной неопределенности;

- расчет статистических показателей результатов сличений для каждого участника;

- интерпретацию статистических показателей для оценки результатов участников.

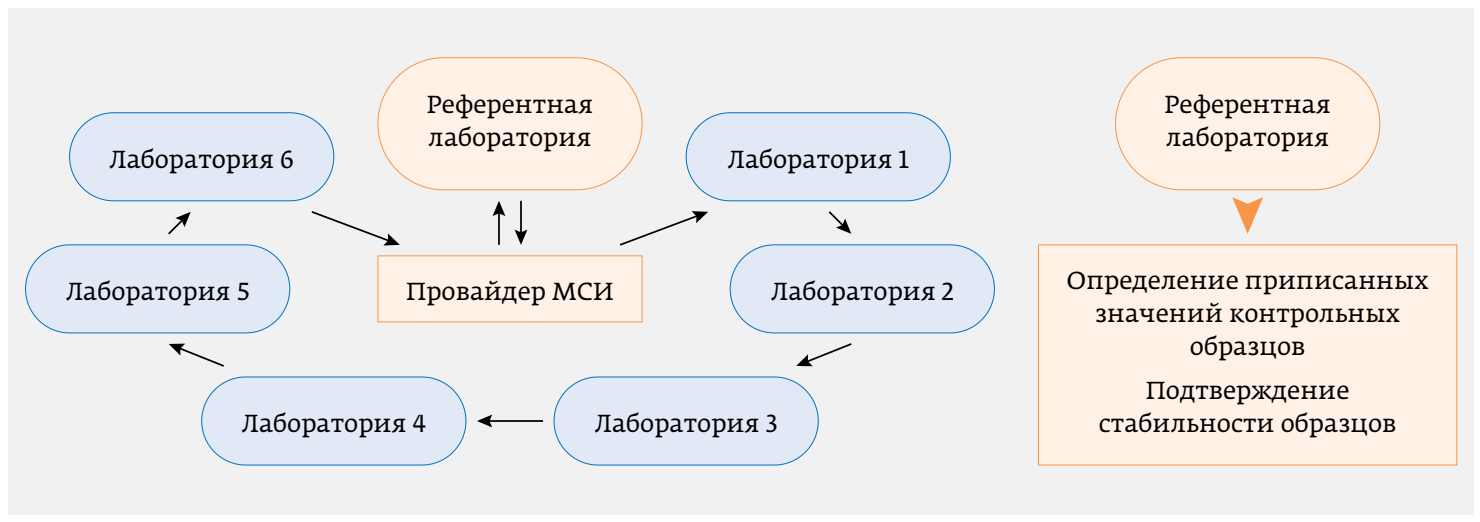

Рис. 2.

Последовательная схема проведения МСИ результатов калибровки электроизмерительных приборов 
В качестве приписанных значений контрольных образцов использовались значения, полученные при их калибровке в референтной лаборатории с помощью многофункционального калибратора Fluke 5522A, аттестованного в качестве эталона единиц электрических величин. Контрольные точки определяются методиками калибровки, разработанными провайдером сличений. Определение расширенной неопределенности приписанных значений также осуществлялось по методикам калибровки.

В качестве статистического показателя для оценки результатов участников выбран показатель $E_{n}$ по пункту 7.5 ГОСТ Р ИСО 13528-2010. Для каждого окончательного результата измерения значение $E_{n}$ рассчитывается по формуле:

$$
E_{n}=\frac{x-X}{\sqrt{U_{\text {lab }}^{2}+U_{\text {ref }}^{2}}},
$$

где: $\boldsymbol{x}$ - значение, определенное в лаборатории-участнице;

$\mathrm{X}$ - приписанное значение, определенное в экспертной лаборатории;

$\mathrm{U}_{\text {ref }}$ - расширенная неопределенность X;

$\mathrm{U}_{\text {lab }}$ - расширенная неопреде-

ленность результата лаборатории-участницы х.

При трактовке показателя $\mathrm{E}_{n}$ устанавливались следующие уровни оценки: если $\left|E_{n}\right|<1$ - результат лаборатории-участницы признается удовлетворительным; если $\left|E_{n}\right| \geq 1$ - результат лаборатории-участницы признается неудовлетворительным.

Пилотный проект был проведен в полном соответствии с требованиями ГОСТ ISO/IEC17043-2013. Отчеты по каждому из двух раундов мСИ содержат большое количество таблиц и графиков. В каждой из участвующих в сличениях лабораторий были проведены серии измерений в 40 точках для мультиметра и 175 точках - для стрелочного прибора. Большинство графиков имеет вид, аналогичный представленному на рис. 3 и 4. Сплошной

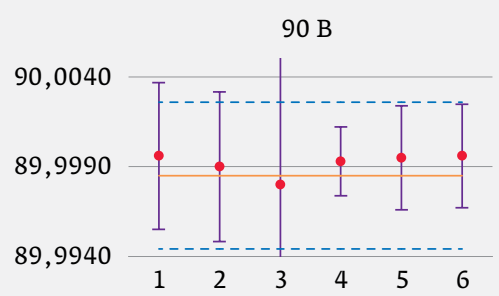

а) напряжение постоянного тока

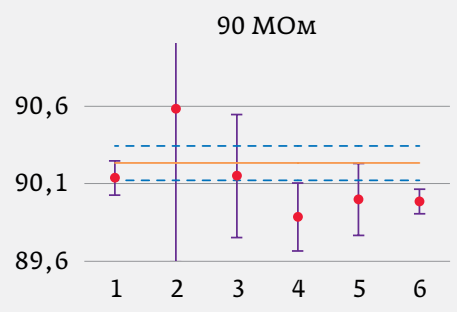

в) электрическое сопротивление
90 мB (50 кГц)

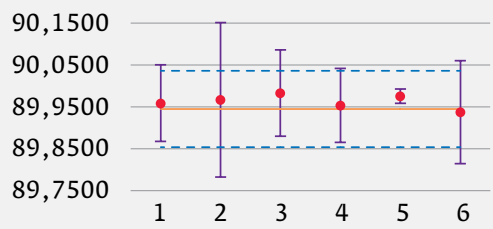

б) напряжение переменного тока

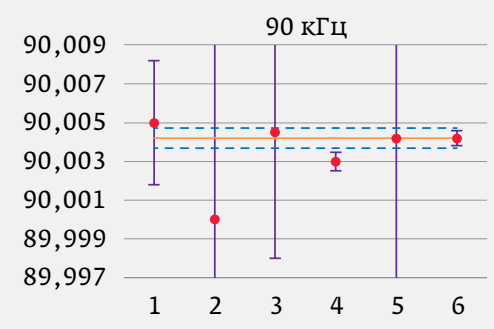

г) частота
Рис. 3. Примеры результатов сличений показаний цифрового мультиметра

горизонтальной линией обозначены приписанные значения показателей, пунктирными линиями - расширенная неопределенность приписанного значения. Красными точками отмечены значения, полученные лабораториями-участницами, вертикальные отрезки показывают расширенные неопределенности для этих значений.

Все участники МСИ подтвердили свою квалификацию в подавляющем большинстве точек измерений. Наличие отклонений показателя больше критического при

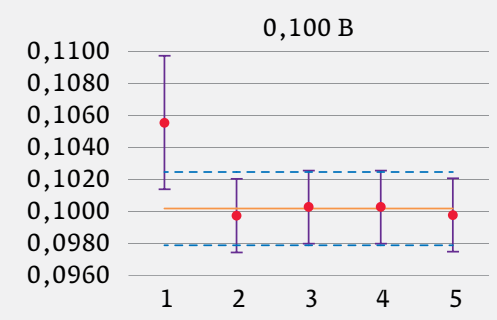

а) напряжение постоянного тока

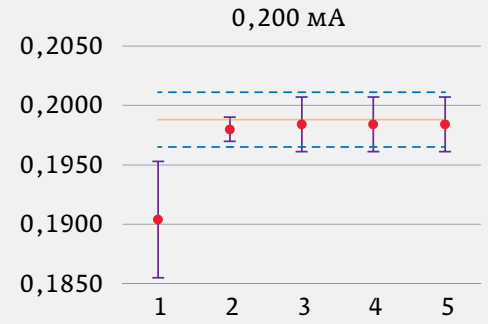

в) сила постоянного тока

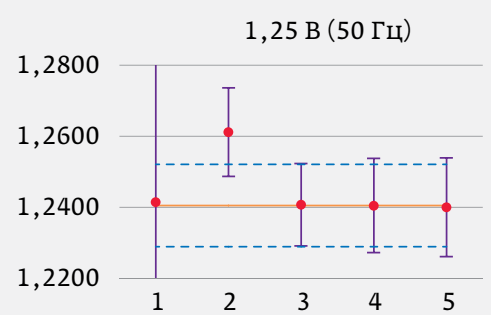

б) напряжение переменного тока

$1,000 \mathrm{MA}(50$ Гц)

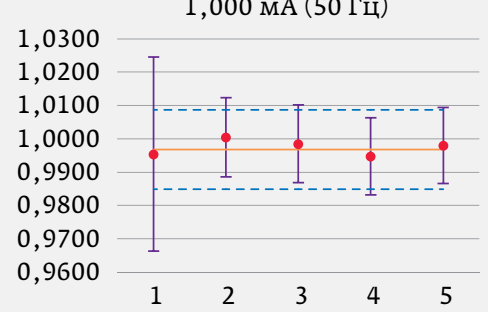

г) сила переменного тока
Рис. 4. Примеры результатов сличений показаний многофункционального стрелочного прибора 
калибровке цифрового мультиметра наблюдалось в 2\% всех измерений; для комбинированного прибора Ц4352 доля неудовлетворительных результатов во всем раунде сличений не превысила 1\%. Иханализ показал, что они могли быть результатами неправильных отсчетов показаний, их регистрации, ошибками оценки неопределенности Были даны рекомендации по контрольным измерениям в соответствующих точках калибровки. Состоялось обсуждение полученных результатов с участниками Мси.

\section{ПЕРСПЕКТИВЫ РАЗВИТИЯ РАБОТ ПО МСИ}

Одним из основных мотивов развития МСИ для метрологических лабораторий является действующее законодательство в области аккредитации. Факт участия лаборатории в МсИ становится показателем качества работы лаборатории при подтверждении компетентности.

Все большее значение приобретает участие в МСИ как средство самоконтроля, которое позволяет выявить проблемы у каждого участника сличений и провести соответствующие предупреждающие и корректирующие действия. Например, описанный пилотный эксперимент показал наличие отклонений больше критических при оценке погрешностей в хорошо оснащенных лабораториях с опытным персоналом для освоенных в практике поверки и калибровки приборов. Очевидно, что доля ошибок для уникальных приборов и методик калибровки, требующих специальной подготовки персонала и учета внешних влияний, будет выше, чем в проведенном эксперименте. Поэтому можно признать проведение МСИ эффективным способом проверки лабораторий, выполняющих калибровку средств измерений.

При этом важно отметить, что каждый участник, используя рекомендации провайдера, сам проводит оценку полученных результатов статистического анализа и их критичности для дальнейшей деятельности. Если для одного участника возможно существование допустимого процента неудовлетворительных результатов, то для более точных и ответственных производств все полученные результаты должны быть положительными. Регулярное участие лаборатории в МСИ позволяет получить оценку качества выполненных измерений и подтвердить как сходимость, так и воспроизводимость результатов измерений при калибровке.

В связи с широким развитием поверочных работ для отечественных предприятий актуален вопрос о целесообразности проведения МСИ результатов поверки средств измерений. На результаты измерений при поверке оказывают влияние все те же факторы, которые определяют правильность и достоверность измерений при калибровке. Основным отличием между МсИ для поверочных и калибровочных лабораторий является вид данных, полученных от лаборатории-участницы и используемых для статистического анализа.
Как при поверке средств измерений, таки при их калибровке основным определяемым параметром является погрешность средства измерений. При проведении МСИ в любых метрологических лабораториях рассматриваемый показатель контрольного прибора - оценка его погрешности. Соответственно, показателем компетентности участника является функционал разности оценки погрешности в лаборатории-участнице и ее приписанного значения, определенного в референтной лаборатории. Это делает актуальным проведение МСИ не только для калибровочных лабораторий, но и для организаций, выполняющих поверку средств измерений в соответствии с действующим законодательством. Применение в рассмотренном примере МСИ показателя $E_{n}$, учитывающего неопределенности калибровки, не должно смущать поверителей. В распоряжении провайдеров МСИ имеется целый арсенал показателей оценки компетентности, в том числе без учета неопределенности. Например, из числа рекомендуемых ГOCT ISO / IEC17043-2013 можно выбрать в качестве статистики Z-показатель:

$$
Z=\frac{x-X}{\hat{\sigma}}
$$

где $\hat{\sigma}$ - стандартное отклонение оценки квалификации, принимаемое участниками сличений для достижения поставленных целей.

Следовательно, обязательным общим требованием к процедуре МСИ для поверочных и калибровочных лабораторий является определение погрешности· $\mathrm{KOH}^{-}$ трольного прибора в соответствии с применяемой методикой в лаборатории-участнице и в референтной лаборатории

Целесообразность проведения МСИ необязательно увязывать с оценкой и подтверждением компетентности метрологических лабораторий в Национальной системе аккредитации или с самоконтролем результатов деятельности по поверке и калибровке средств измерений. Они также могут служить объективным способом проверки правильности измерений параметров изделий на входном контроле, при технологических процессах изготовления и испытаниях продукции. Такая задача может быть актуальной для предприятий, осваивающих новую продукцию, внедряющих новые методы и средства контроля, когда вопрос качества измерений стоит достаточно остро.

Уже сейчас крупные корпорации активно внедряют мСИ результатов поверки и измерений между лабораториями своих предприятий. Подобная практика помогает оценивать качество работы лабораторий и поддерживать его на одном уровне, проводя корректирующие или предупреждающие действия при выявлении расхождений результатов измерений. 
МСИ могут выполнять важную роль контроля качества измерений важнейших параметров продукции, прежде всего основных показателей назначения. Довольно часто при таких измерениях используются сложные технические системы и устройства с измерительными функциями, для которых метрологическое обеспечение представляет собой сложную организационную и техническую задачу. Организация межлабораторных сличений результатов измерений внешне похожа на МСИ для испытательных лабораторий. Аналогично последним необходимо выбрать контрольный образец и его параметры, выбрать и определить приписанные значения. Однако статистический анализ результатов в данном случае должен основываться на оценках и анализе метрологических характеристик используемых технических средств, методических составляющих погрешностей.

\section{выводы}

В практике деятельности метрологических подразделений, занимающихся поверкой или калибровкой средств измерений, бывают случаи, когда результаты их работы подвергаются сомнениям или даже критике. Именно это является причиной внимания к межлабораторным сличениям результатов калибровки и поверки средств измерений со стороны аккредитующих и других организаций, заинтересованных в квалификации лабораторий. Наглядность результатов, полученных при сличениях, их объективность и возможность контроля влияния факторов, которые сложно определить при обычных аудитах, проводимых органами по аккредитации, заставляют уделять этому способу контроля качества большое внимание.

Полученные результаты пилотных раундов МСИ говорят о целесообразности широкого участия в них предприятий промышленности. Особое внимание предприятиям стоит уделить планированию участия в МСИ для областей и средств измерений, используемых при контроле наиболее ответственных параметров продукции и услуг.

Проведение МСИ может быть востребовано для различных задач метрологических служб, таких как оценка результатов поверки и калибровки средств измерений, поддержание единого уровня точности в лабораториях корпораций, контроль результатов измерений наиболее ответственных параметров продукции, оценка применимости новых методик, средств измерений и технических средств. Регулярность проведения МсИ открывает возможности для непрерывного мониторинга качества метрологических работ, совершенствования порядка проведения работ и внедрения удобных схем сличений с минимальными издержками. 